\title{
Signature of Electron-Plasmon Quantum Kinetics in GaAs
}

\author{
Q. T. Vu and H. Haug \\ Institut für Theoretische Physik, J. W. Goethe-Universität Frankfurt, Robert-Mayer-Straße 8, 60054 Frankfurt am Main, Germany
}

\author{
W. A. Hügel, S. Chatterjee, and M. Wegener \\ Institut für Angewandte Physik, Universität Karlsruhe (TH), Kaiserstraße 12, 76128 Karlsruhe, Germany
}

(Received 28 June 2000)

\begin{abstract}
We predict a carrier-density dependent oscillation, which is superimposed on the decay of the coherent control photon echo signal of a semiconductor. It reflects the oscillatory transfer of excitation back and forth between electrons and a mixed plasmon-phonon mode. This signature provides obvious and unique evidence for the finite duration of the interaction process, i.e., evidence for the collective Coulomb quantum kinetics. The theoretical predictions for the model semiconductor GaAs are reproduced in corresponding experiments.
\end{abstract}

PACS numbers: 78.47.+p, 42.50.Md, 42.65.Re, 63.20.Kr

Dissipation and relaxation processes in solid state physics can often be thought of as a transfer of excitation from a part of the problem, "the system," to the rest, "the bath." For the well-studied example of electron-phonon interaction in semiconductors, this separation into system and bath is obvious. Yet, it has only been recently that quantum kinetics has revealed the rich real-time quantum mechanical details of this transfer process [1-3]. The main new point of quantum kinetics - as compared to semiclassical Boltzmann kinetics - is that two time scales are involved: the mean time between collisions and the duration of an interaction process. One signature of this finite duration is a coherent oscillation which results from a combined electron-phonon coherence, i.e., the excitation oscillates back and forth between system and bath for a short time span, the memory time. The analog of this process for a molecule or a quantum dot, where one has discrete states rather than energy bands, would be a quantum beating between an electronic state and its vibrational sideband [4].

These concepts of quantum kinetics reach far beyond solid state physics. They are currently also discussed for heavy-ion collisions, for nonequilibrium nuclear matter, and for plasmas in strong electromagnetic fields (for a recent survey, see, e.g., Ref. [5]).

For the case of electron-electron interaction in semiconductors, a separation of the complete problem into system and bath contributions is not obvious at all. As a result, many groups have addressed various aspects of this fundamental problem in the dilute limit [6-8], where memory effects due to biexcitonic correlations do occur indeed [8], as well as for elevated carrier densities [9-15], where plasma screening becomes important. Intuitively, one would expect that the collective excitation of the electron gas, the plasmon, could play the role of the phonon in the above example. In analogy to the electron-phonon quantum kinetics, one should then be able to observe an oscillation corresponding to the transfer of excitation back and forth between individual electrons (the system) and the collective mode of the electrons, i.e., the plasmons (the bath). This combined electron-plasmon coherence, which must not be confused with coherent plasmons [16], has neither been observed experimentally, nor has any realistic theoretical suggestion been made as to how it could be measured.

In this paper, we first show theoretically that a coherent control photon echo experiment is suitable to identify this process. As a signature, we predict a carrier-density dependent oscillation which is superimposed on the photon echo signal and which is due to a mixed plasmon-phonon mode. Corresponding experiments, which agree well with this prediction, are also presented.

Why has this signature not been observed before? It is clear that one has to get into a regime of carrier densities in which the plasmon energy $\hbar \omega_{\mathrm{pl}}$ is comparable to or larger than the longitudinal optical (LO) phonon energy $\hbar \omega_{\text {LO }}$. For the case of the well-defined model semiconductor GaAs, where one has $\hbar \omega_{\mathrm{LO}}=36 \mathrm{meV}$ and $\hbar \omega_{\mathrm{pl}}(0)=$ $\hbar \sqrt{4 \pi n_{\mathrm{eh}} e^{2} /\left[\varepsilon(0) m^{*}\right]}$ (at zero plasmon momentum $q$, cgs units), with static dielectric constant $\varepsilon(0)$ and reduced effective mass $m^{*}$, this crossing, $\hbar \omega_{\mathrm{LO}}=\hbar \omega_{\mathrm{pl}}(0)$, would happen at a carrier density around $n_{\mathrm{eh}}=10^{18} \mathrm{~cm}^{-3}$. As plasmons and LO phonons interact via Coulomb interaction, one expects an anticrossing behavior, corresponding to a mixed plasmon-phonon mode. Under these conditions, neither we [14] nor others $[9,15]$ have seen clear signs of oscillations in photon echo experiments (or in any other technique). This is not surprising in view of the fact that the overall decay times under these conditions are in the range of 10 to 20 femtoseconds $[9,14,15]$, which does not strongly favor the observation of oscillations with expected periods in the range of 50 to 100 femtoseconds. A way out of this dilemma is the tailoring of the initial state wave function by means of coherent control $[17,18]$, which helps to enhance the visibility of hidden structures. Here, we employ a simple and known form of coherent control, where a pair of phase-locked femtosecond pulses, 1 and $1^{\prime}$, with optical wave vector $\vec{K}_{1}=\vec{K}_{1^{\prime}}$ and corresponding 
time delay $t_{11^{\prime}}=t_{1}-t_{1^{\prime}}$, excites the semiconductor [19]. We evaluate the photon echo signal with optical wave vector $2 \vec{K}_{2}-\vec{K}_{1}$, induced by a second pulse, 2 , with optical wave vector $\vec{K}_{2}$ and time delay $t_{21^{\prime}}=t_{2}-t_{1^{\prime}}$.

Description of the theory.-The effective screened potential $W_{q}(1,2)$ in the time-dependent random phase approximations (RPA) obeys the equation

$$
W_{q}(1,2)=W_{q}^{0}(1,2)+W_{q}^{0}(1,3) L_{q}(3,4) W_{q}(4,2),
$$

where 1,2 , etc., are time arguments on the Keldysh contour, repeated indices have to be integrated over the contour, and $q$ is the wave number. The RPA polarization function is given by $L_{q}(1,2)=-2 i \hbar \sum_{\vec{k}} G_{\vec{k}}(1,2) \times$ $G_{\vec{k}-\vec{q}}(2,1) . \quad G$ is the carrier Green functions. In the considered two-band model, these functions are matrices in the band index having also off-diagonal elements which are induced by the coherent light pulses. The LO-phonon mediated interaction and the Coulomb interaction are treated on the same footing, i.e., $W_{q}^{0}(1,2)=V_{q} \delta(1,2)+$ $g_{q}^{2} D_{q}(1,2)$, where $V_{q}$ is the bare Coulomb potential and $D_{q}(1,2)$ is the propagator of the phonons which we approximate as a thermal bath. The matrix elements $g_{q}$ describe LO-phonon-electron Fröhlich interaction in a polar semiconductor. The two resonances in the effective potential describe the time dependence of the two branches of the mixed phonon-plasmon spectrum. As described in more detail in Ref. [20], Eq. (1) has to be disentangled by making a transition from the Keldysh-contour Green functions to its real-time components. As the RPA is not a conserving approximation, Eq. (1) generally contains a $q \rightarrow 0$ divergence. However, in the one-time version of quantum kinetics in which the two-time propagators are expressed by the so-called generalized Kadanoff-Baym approximation [21] in terms of one-time density matrices, the resulting scattering rates

$$
\left.\frac{\partial \rho_{k}}{\partial t}\right|_{\text {scatt }}=-\frac{1}{\hbar} \int_{-\infty}^{t} d t^{\prime}\left[\Sigma_{k}^{>}\left(t, t^{\prime}\right) G_{k}^{<}\left(t^{\prime}, t\right)-\Sigma_{k}^{<}\left(t, t^{\prime}\right) G_{k}^{>}\left(t^{\prime}, t\right)-G_{k}^{>}\left(t, t^{\prime}\right) \Sigma_{k}^{<}\left(t^{\prime}, t\right)+G_{k}^{<}\left(t, t^{\prime}\right) \Sigma_{k}^{>}\left(t^{\prime}, t\right)\right]
$$

are finite, when calculated in the self-consistent so-called $G W$ approximation [22]. The unitary part of the time evolution of $\rho(t)$ is given by the semiconductor Bloch equations describing electrons in a two-band semiconductor under the influence of the classical light field and the Coulomb exchange interaction. For more details of the theory we refer the reader to Ref. [20]. A projection technique [23] is used to calculate the photon echo signal propagating with optical wave vector $2 \vec{K}_{2}-\vec{K}_{1}$.

The parameters of the calculations are as follows. The optical pulses, $1,1^{\prime}$, and 2 , arrive at times $t_{1}, t_{1^{\prime}}$, and $t_{2}$, they all have a $\operatorname{sech}^{2}(t)$ shape and a duration (full width at half maximum of the intensity) of $11 \mathrm{fs}$. Furthermore, all three pulses have the same intensity and their spectrum is centered around $30 \mathrm{meV}$ above the band gap. The room temperature GaAs band gap energy is $E_{g}=$ $1425 \mathrm{meV}$, the electron and (heavy-) hole effective masses are $m_{\mathrm{e}}=0.069 m_{0}$ and $m_{\mathrm{h}}=0.50 m_{0}$, respectively. $m_{0}$ is the free electron mass. The dielectric constants are $\varepsilon(0)=12.9$ and $\varepsilon(\infty)=10.9$, the exciton Rydberg energy is $E_{\mathrm{R}}=4.2 \mathrm{meV}$. The total carrier densities $n_{\mathrm{eh}}$ given in the following are defined as the incoherent sum of the individual one-pulse carrier densities of the three incident pulses $1,1^{\prime}$, and 2 . In this way we can define carrier densities in a coherent control geometry unambiguously and consistently for both theory and experiment. This allows for a direct comparison. The interpretation of the sodefined value for $n_{\text {eh }}$ does, however, require some caution because the actually excited carrier density also depends on the time delay $t_{11^{\prime}}$ (and on $t_{21^{\prime}}$ ), as we will explain below.

Figure 1(a) shows the calculated behavior of the photon echo signal spectra versus time delay $t_{11^{\prime}}$. In (b), two selected traces versus time delay $t_{21^{\prime}}$ are shown, which represent the two limits of no oscillations $\left(t_{11^{\prime}}=-20.8 \mathrm{fs}\right)$ and most pronounced oscillations $\left(t_{11^{\prime}}=-22.0 \mathrm{fs}\right)$. The

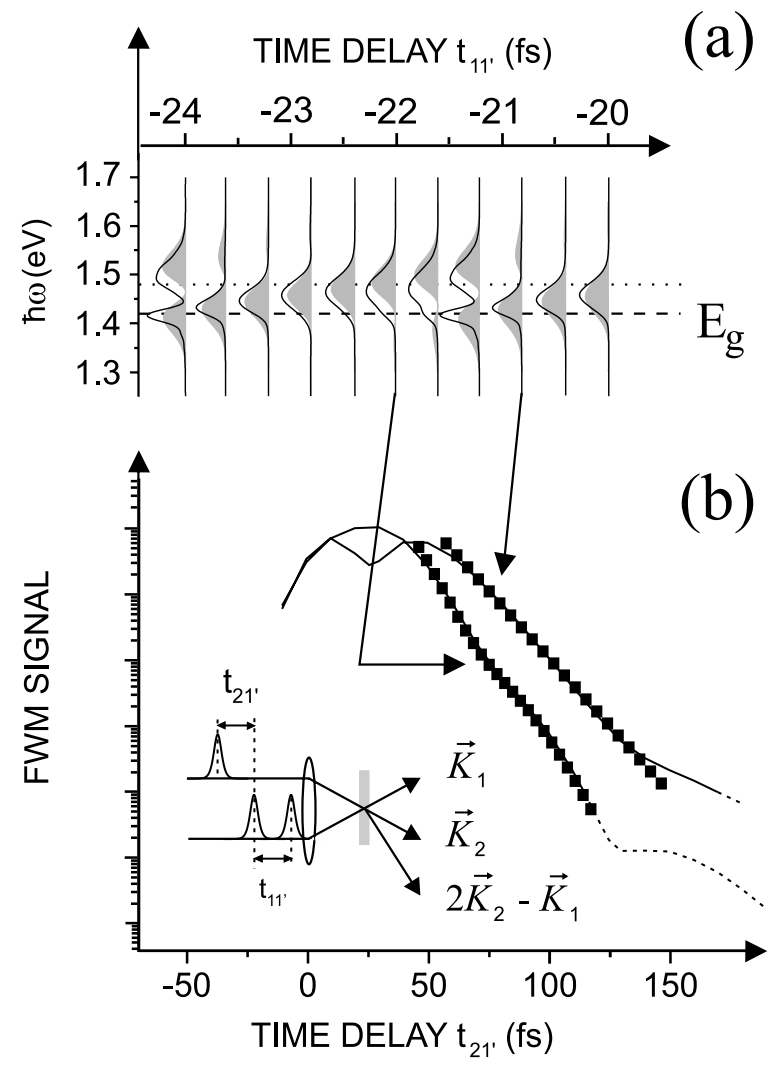

FIG. 1. Examples of calculated results: (a) Spectra (individually normalized) of the phase-locked pulse-pair (shaded) and the photon echo signal at $t_{21^{\prime}}=30 \mathrm{fs}$, and (b) two selected traces versus time delay $t_{21^{\prime}}$ for $t_{11^{\prime}}=-20.8 \mathrm{fs}$ and $t_{11^{\prime}}=-22.0 \mathrm{fs}$ plotted on the same scale. The symbols in (b) are the result of simple fits. Bulk GaAs at $T=300 \mathrm{~K}$ excited by $11 \mathrm{fs}$ pulses, $n_{\mathrm{eh}}=1.0 \times 10^{18} \mathrm{~cm}^{-3}$. The oscillation period under these conditions is $60 \mathrm{fs}$. The inset illustrates the geometry. 
signal inside the experimentally accessible range of 4 orders of magnitude is shown by full lines, outside of this range by dashed lines. The data strongly suggest that the behavior can be understood qualitatively as a quantum beating between the band gap and the plasmon sideband of the band gap [see dotted lines in Fig. 1(a)]: The plasmon sideband has a weaker optical oscillator strength than the band gap. Thus, if we suppress the band gap by corresponding excitation, the two oscillators become effectively comparable in strength, and pronounced oscillations are expected. This is the case for $t_{11^{\prime}}=-22.0 \mathrm{fs}$ [Fig. 1(a)]. On the other hand, when the plasmon sideband is not excited by the spectrum of the pulse pair, which is the case for $t_{11^{\prime}}=-20.8 \mathrm{fs}$, no oscillations are observed. However, the FWM spectra show that the behavior is more intricate. For the case of pronounced oscillations in the $t_{21^{\prime}}$ domain $\left(t_{11^{\prime}}=-22.0 \mathrm{fs}\right)$, the FWM spectrum is a featureless broad maximum, indicating no oscillations in the real-time domain.

Figure 2 shows the experiment corresponding to Fig. 1. The optical pulses have a duration of $13 \mathrm{fs}$; all other parameters correspond to what has been stated above, the antireflection-coated, $600 \mathrm{~nm}$ thin bulk GaAs sample is identical to Refs. $[1,3,14]$. The values for $t_{11^{\prime}}$ are measured absolutely (not only relatively) using the "Pancharatnam screw" [24] (with an error of $\pm 0.05 \mathrm{fs}$ ). Beside some shift in the absolute position of $t_{21^{\prime}}=0$, the agreement between theory (Fig. 1) and experiment (Fig. 2) is good.

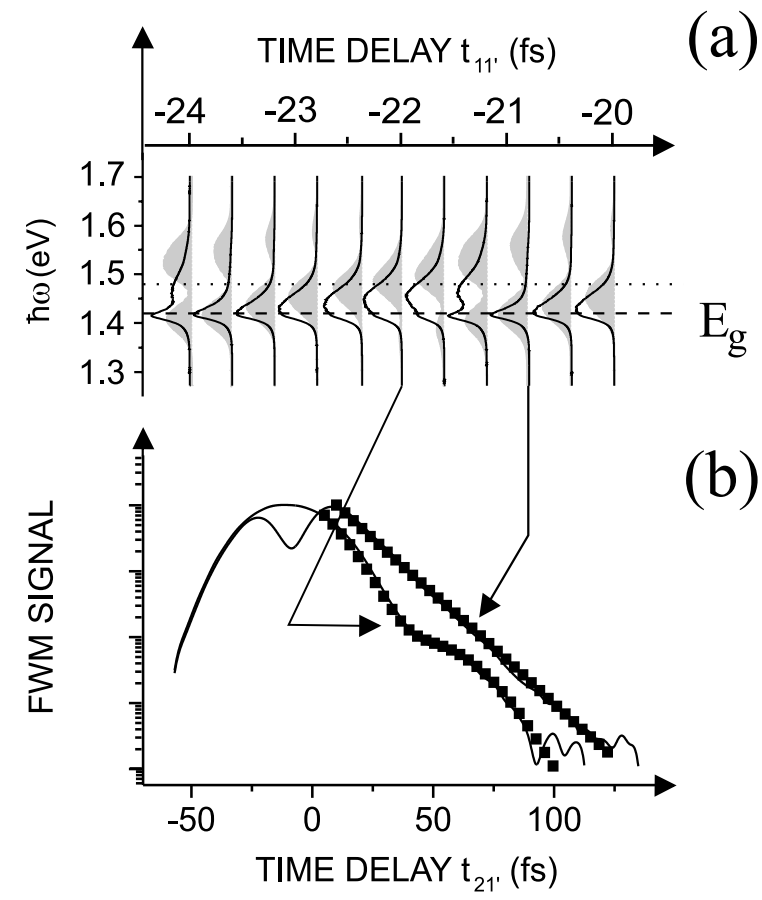

FIG. 2. Examples of experimental results presented as Fig. 1 (theory). (a) $t_{21^{\prime}}=0 \mathrm{fs}$, the symbols in (b) are the result of simple fits. Bulk GaAs at $T=300 \mathrm{~K}$ excited by $13 \mathrm{fs}$ pulses, $n_{\mathrm{eh}}=5.4 \times 10^{17} \mathrm{~cm}^{-3}$. The oscillation period under these conditions is $68 \mathrm{fs}$.
Figure 3 shows the decay time $\tau$ of the FWM signal versus time delay $t_{11^{\prime}}$, which is obtained from fits to the data similar to the ones shown in Figs. 1 and 2. For those optical transitions, for which $t_{11^{\prime}}$ corresponds to destructive interference, the carriers are excited by pulse 1 and coherently reemitted by pulse $1^{\prime}$. This leads to a modulation of the carrier density [17] versus $t_{11^{\prime}}$, which results in an obvious modulation of the carrier-carrier scattering time. From this alone, one would expect a symmetric modulation of $\tau=\tau\left(t_{11^{\prime}}\right)$ and not the asymmetric sawtooth shape shown in Fig. 3. This particular line shape is due to the fact that not only the carrier density but also the shape of the carrier distribution is coherently modulated with time delay $t_{11^{\prime}}$. Note that, again, theory [Fig. 3(a)] and experiment [Figs. 3(b) and 3(c)] agree quite well.

Figure 4 summarizes the oscillation period versus carrier density $n_{\text {eh }}$ as determined from the data of Figs. 1, 2 , and others. The data at very low carrier densities $(T=$ $77 \mathrm{~K})$ are taken from the literature [1,3]. For comparison, we also depict as dashed lines the LO phonon and the plasmon oscillation periods, $2 \pi / \omega_{\mathrm{LO}}$ and $2 \pi / \omega_{\mathrm{pl}}(0)$, respectively. The full horizontal line corresponds to $2 \pi /\left[\omega_{\mathrm{LO}}\left(1+m_{\mathrm{e}} / m_{\mathrm{h}}\right)\right] \approx 98 \mathrm{fs}$. The extra mass factor occurs, because two conduction band states which are connected by the exchange of one longitudinal optical quantum $\hbar \omega_{\text {LO }}$ lead to a larger difference of the two associated optical transition energies, thus to beating

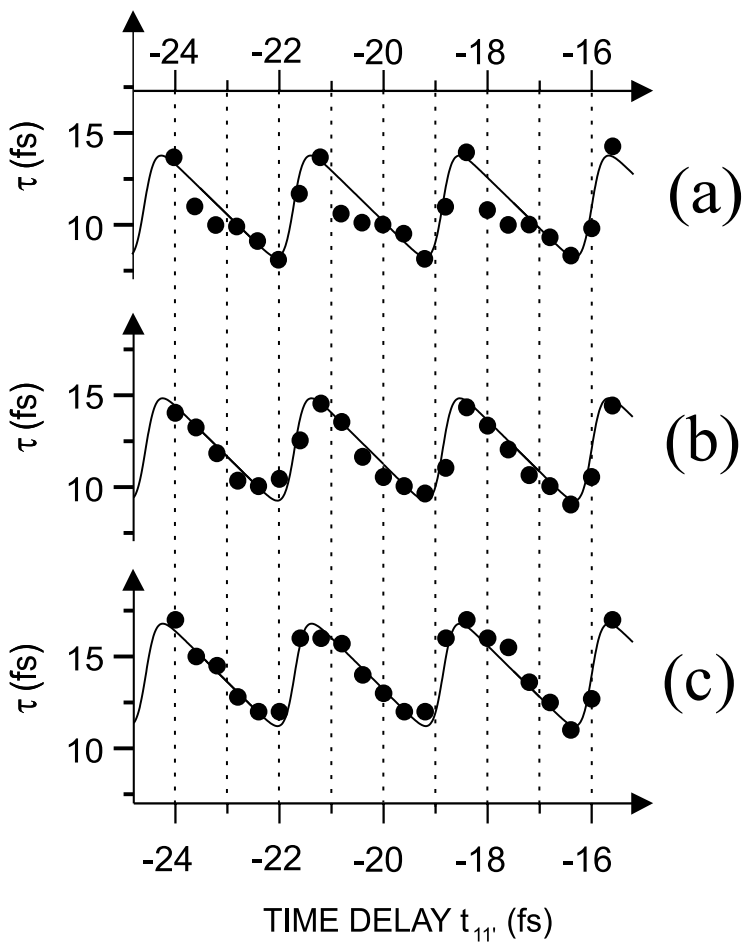

FIG. 3. Decay time $\tau$ of the photon echo signal versus time delay $t_{11^{\prime}}$ as determined from fits to the data [Figs. 1(b) and 2(b)]. (a) theory, $n_{\mathrm{eh}}=1.0 \times 10^{18} \mathrm{~cm}^{-3}$, (b) experiment, $n_{\mathrm{eh}}=1.1 \times 10^{18} \mathrm{~cm}^{-3}$, (c) experiment, $n_{\mathrm{eh}}=5.4 \times$ $10^{17} \mathrm{~cm}^{-3}$. The full curves are guides to the eye. 


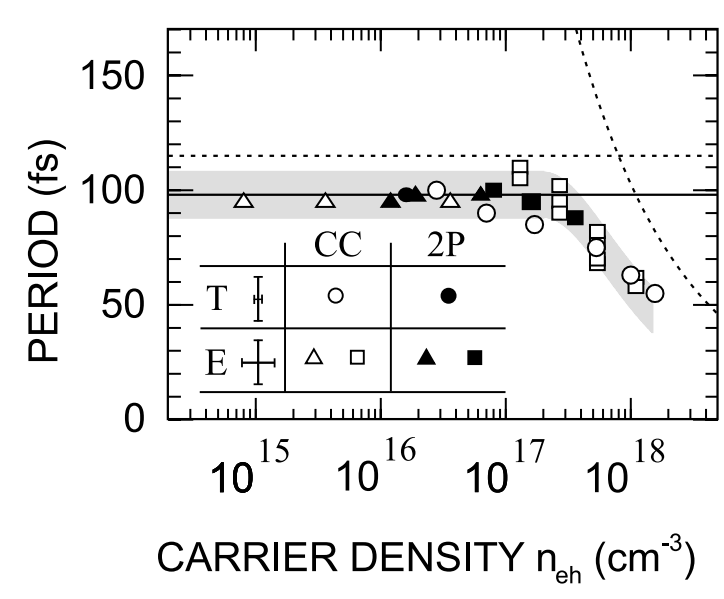

FIG. 4. Theory (T) and experiment (E): Oscillation period versus carrier density $n_{\text {eh }}$. Filled symbols are two-pulse (2P) photon echo results, open symbols are from coherent control (CC), see legend, with error bars as indicated. Triangles correspond to experiments at $77 \mathrm{~K}$, squares to experiments at $300 \mathrm{~K}$ sample temperature. The shaded area is a guide to the eye. The two dashed curves correspond to the bare LO-phonon and the plasmon oscillation period, $2 \pi / \omega_{\mathrm{LO}}$ and $2 \pi / \omega_{\mathrm{pl}}(0)$, respectively.

faster than $\omega_{\mathrm{LO}}$, due to the finite curvature of the valence band [1].

It is obvious that the observed oscillation period becomes shorter for carrier densities exceeding several $10^{17} \mathrm{~cm}^{-3}$. This density-dependent oscillation is the anticipated signature of electron-plasmon quantum kinetics. Actually, in this regime, one has a mixed plasmon-phonon mode. The corresponding avoided crossing occurs at smaller $n_{\mathrm{eh}}$ than one would expect from the plasma frequency at zero momentum transfer, $\hbar \omega_{\mathrm{pl}}(0)$. An inspection of the scattering rates and their wave number dependence shows that the effective interaction is strongest for the exchange of a quantum of the upper branch of the plasmon-phonon mode at finite momentum transfer $q=\mathcal{O}\left(a_{0}^{-1}\right)$, where $a_{0}$ is the exciton Bohr radius. This, together with the parabolic dispersion $\omega_{\mathrm{pl}}(q) \simeq$ $\omega_{\mathrm{pl}}(0)\left(1+\frac{1}{2} q^{2} / \kappa^{2}\right)$, where $\kappa$ is the inverse screening length, explains why the observed oscillations have periods less than $2 \pi /\left[\omega_{\mathrm{pl}}(0)\left(1+m_{\mathrm{e}} / m_{\mathrm{h}}\right)\right]$. The origin of the extra mass factor is analogous to the phonons. This inspection also shows that the lower branch of the plasmon-phonon mode has negligible strength for the scattering.

In conclusion, in this article, we have theoretically predicted a signature of electron-plasmon quantum kinetics, which is consistent with corresponding coherent control photon echo experiments with 13 fs pulses on bulk GaAs at $T=300 \mathrm{~K}$, also presented here. For carrier densities around $10^{18} \mathrm{~cm}^{-3}$, this signature is a coherent oscillation, the period of which follows the upper branch of a mixed plasmon-phonon mode. This observation highlights the analogies between the relatively simple electron-phonon and the collective electron-electron quantum kinetics.

This collaboration has been supported by the DFG Schwerpunktprogramm Quantenkohärenz in Halbleitern. M.W. gratefully acknowledges support by the DFG Leibniz award. We thank W. Stolz for the high quality sample.

[1] L. Bányai et al., Phys. Rev. Lett. 75, 2188 (1995).

[2] C. Fürst et al., Phys. Rev. Lett. 78, 3733 (1997).

[3] M. U. Wehner et al., Phys. Rev. Lett. 80, 1992 (1998).

[4] R. W. Schoenlein et al., Phys. Rev. Lett. 70, 1014 (1993).

[5] Progress in Non-Equilibrium Green's Functions, edited by M. Bonitz (World Scientific, Singapore, to be published).

[6] P. Kner et al., Phys. Rev. Lett. 78, 1319 (1997).

[7] G. Bartels et al., Phys. Rev. Lett. 81, 5880 (1998).

[8] C. Sieh et al., Phys. Rev. Lett. 82, 3112 (1999).

[9] P. C. Becker et al., Phys. Rev. Lett. 61, 1647 (1988).

[10] T. Elsaesser et al., Phys. Rev. Lett. 66, 1757 (1991).

[11] D. C. Scott et al., Phys. Rev. Lett. 69, 347 (1992).

[12] F. X. Camescasse et al., Phys. Rev. Lett. 77, 5429 (1996).

[13] L. Bányai et al., Phys. Rev. Lett. 81, 882 (1998).

[14] W. A. Hügel et al., Phys. Rev. Lett. 83, 3313 (1999).

[15] S. Arlt et al., Phys. Rev. B 59, 14860 (1999).

[16] W. Sha et al., Phys. Rev. Lett. 74, 4273 (1995). The observation of coherent plasmons in this reference corresponds to a macroscopic real-space oscillation of charges in the classical sense. In our work, this is not the case. Here, the quantum mechanical fluctuations of the plasmon lead to the oscillations,

[17] A. P. Heberle et al., Phys. Rev. Lett. 75, 2598 (1996).

[18] X. Hu and W. Pötz, Phys. Rev. Lett. 82, 3116 (1999).

[19] Instead of using a pair of phase-locked pulses one could alternatively use a pulse shaper to influence both amplitude and phase of the pulse spectrum.

[20] Q. T. Vu and H. Haug, Phys. Rev. B (to be published).

[21] P. Lipavský et al., Phys. Rev. B 34, 6933 (1986).

[22] P. Gartner et al., Phys. Rev. B (to be published).

[23] H. Haug and A.P. Jauho, Quantum Kinetics in Transport and Optics of Semiconductors (Springer, Berlin, 1996).

[24] M. U. Wehner et al., Opt. Lett. 22, 1455 (1997). 